\title{
Osteoarticular Complications Related to HIV Infection and Highly Active Antiretroviral Therapy
}

\author{
Ana Lúcia Lei Munhoz Lima ${ }^{1}$, Arnaldo Valdir Zumiotti ${ }^{2}$, Gilberto Luis Camanho ${ }^{2}$, Eduardo Benegas ${ }^{1}$, \\ Alexandre Leme Godoy dos Santos ${ }^{1}$, Caio Oliveira D'Elia ${ }^{1}$ and Priscila Rosalba Domingos Oliveira ${ }^{1}$ \\ ${ }^{1}$ Institute of Orthopedics and Traumatology, Clinics Hospital, School of Medicine of the University of São Paulo (FMUSP); ${ }^{2}$ Department of \\ Orthopedics and Traumatology of FMUSP; São Paulo, SP, Brazil
}

\begin{abstract}
With the significant increase in life expectancy for HIV-infected patients in the era of high potency antiretroviral therapy, major metabolic changes have been observed due to the prolonged period of the viral infection and the treatment itself. Osteoarticular changes resulting from these processes are mainly reported in long term HIVinfected patients receiving high potency antiretroviral therapy and include osteopenia/osteoporosis, osteonecrosis, carpal tunnel syndrome and adhesive capsulitis of the shoulder.
\end{abstract}

Key-Words: HIV, acquired immunodeficiency syndrome, osteoarticular, antiretroviral agents.

With the significant increase in life expectancy for HIVinfected patients in the era of highly active antiretroviral therapy, a few consequences have been observed concerning the extended time to viral infection and therapy. Metabolic consequences occurring within this context have been explored in the literature, mainly concerning the lipodystrophic syndrome. The increasing observation of osteoarticular changes in these patients is currently the object of more detailed studies for the purpose of detecting their possible causes and a more appropriate therapeutic approach.

Complex metabolic changes in chronic HIV infection and its treatment include the decrease of bone mineralization in a high proportion of patients, as a result of various factors present in the host itself, the virus and the antiretroviral agents. Bone is continuously remodeled due to the synchronism between its formation and resorption, although it can be deregulated during an HIV infection. Bone mineralization decreases cause osteopenia, which may result in osteoporosis.

Osteoarticular changes most frequently reported by HIVinfected patients receiving high potency antiretroviral therapy for long periods include osteopenia/osteoporosis, osteonecrosis, carpal tunnel syndrome and adhesive capsulitis of the shoulder.

\section{Osteopenia/Osteoporosis}

The World Health Organization definitions of osteopenia and osteoporosis are based on bone densitometry results obtained by comparing the ratio between the patient's bone density and its mean value for young adults, and adjusting the results for ethnics and gender [1]. Osteoporosis occurs when this ratio is less than two times the standard deviation, while osteopenia occurs when this ratio is between minus

Received on 22 February 2007; revised 10 July 2007.

Address for correspondence: Dr. Ana Lúcia Munhoz Lima. Rua Dr. Ovídio Pires de Campos, 333, Cerqueira César. Zip code: 05403-010. São Paulo, SP, Brazil. Phone (11) 3069-6921. Fax: 3069-6920. Email: ccih.iot@hcnet.usp.br.

The Brazilian Journal of Infectious Diseases

2007;11(4):426-429. (C) 2007 by The Brazilian Journal of Infectious Diseases and Contexto Publishing. All rights reserved. one time and minus two times the standard deviation [2]. Osteoporosis is considered severe if, additionally to meeting this criterion, the patient has a fracture.

Several studies have shown high prevalence of these changes in HIV-infected patients according to these criteria [14,6-11]. The multiple factors listed as promoters of osteopenia include direct effects of the virus on osteogenic cells; persistent activation of proinflammatory cytokines, mainly TNF- $\alpha$ and interleukin-1; changes in vitamin D metabolism, with deficit of 1,25 di-hydrovitamin $\mathrm{D}$, as well as the involvement of mitochondrial abnormalities related with lactic acidemia and the development of opportunistic diseases [4,5].

Studies on the influence of antiretroviral treatment show that the relative risk is higher when protease inhibitors are used, and indinavir is known to inhibit bone formation, while ritonavir inhibits osteoclast differentiation and function [1,6,7,9-11]. Regarding reverse transcriptase inhibitors, recent reports have linked tenofovir to the occurrence of osteomalacia and the Fanconi syndrome [12]. Other factors can also contribute to accelerate bone loss, such as nutritional deficiency, low serum calcium levels, immobilization, hypogonadism, hyperthyroidism, hyperparathyroidism, renal failure, use of opioids or heroin, use of corticosteroids, postmenopausal period, and alcohol intake higher than $16 \mathrm{~g} /$ day $[1,8]$.

The main steps to prevent osteoporosis include encouragement to practice physical activities and an appropriate diet during the first three life decades, in order to achieve maximum bone mass formation.

Calcium and supplementary vitamin D intake should be part of any therapeutic regimen for osteoporosis. For postmenopausal women, hormone replacement therapy is an important method for preventing osteoporosis. There are basically two classes of drugs, e.g. bone tissue antiresorption agents and bone formation stimulating agents. Examples of antiresorption agents include estrogens, calcitonin and biphosphonates. Sodium fluoride and parathormone are bone formation stimulating agents [1].

\section{Osteonecrosis}

The occurrence of osteonecrosis in HIV-infected patients has been reported since 1990, with progressively increasing 
incidence, higher than that in the general population $[7,8]$. The estimated yearly incidence of symptomatic osteonecrosis in the general population ranged between 0.010 and $0.135 \%$ [13].

Recent studies using magnetic resonance imaging to detect osteonecrosis in HIV-positive patients estimate that the incidence of this condition is approximately $4 \%$. The incidence of bilaterality ranges between 35 and $80 \%$ [13].

Some authors refer to stabilization of osteonecrosis indexes in the general population during the last few years, although this phenomenon is still increasing in the population of HIV-infected patients. The general population presents some known risk factors and conditions associated with the development of osteonecrosis, such as the use of systemic corticosteroids, alcohol abuse, hyperlipidemia, sickle cell anemia, coagulopathies, Gaucher disease, systemic lupus erythematosus, rheumatoid arthritis, hyperuricemia and gout, radiotherapy, obesity, pancreatitis, fracture sequelae, chemotherapy, vasculitis, and smoking. In HIV-infected patients, other factors besides the above mentioned ones are involved in the development of osteonecrosis, such as dyslipidemia, the use of megestrol acetate and anabolizers, testosterone replacement, as well as vasculitis predisposing to intrabone thrombosis due to the presence of anticardiolipin antibodies and protein S deficiency. The antiretroviral treatment itself may be related to increasing development of osteonecrosis $[7,8,13]$.

When diagnosing osteonecrosis, clinical signs should be observed such as the presence of joint pain and range of motion limitations. The most frequently involved joints are hips (unilaterally or bilaterally), knees, ankles, elbows and shoulders $[14,15]$. The following investigation algorithm is proposed in Figure 1.

It is worth noticing that the interval between radiological changes and clinical symptoms may be long, ranging between three and eight years. Ordinary radiographs of the joint have low diagnostic sensitivity in the early onset of the disease. Cystic sclerosis, subchondral radiolucency, bone collapse and degenerative changes in the joints are frequent radiological findings indicating osteonecrosis. Computerized tomography without contrast adds little information to ordinary radiographs. Magnetic resonance imaging is highly sensitive (99\%) and specific in early diagnosis. Bone scintigraphy is not very specific, but it can be used in staging and searching for asymptomatic occult focuses $[14,15]$. Based on clinical and magnetic resonance image findings, it is possible to classify osteonecrosis. FicatArlet staging system is the most used in hip osteonecrosis [16] (Table 1).

The treatment will vary depending on the stage of the disease. In HIV-positive patients it is important to exclude or control risk factors other than the ones of the disease itself and the antiretroviral drug. In oligosymptomatic subjects the treatment may be based on the use of analgesics and non-hormonal anti-inflammatory agents [6].
During the early stages it is possible to use decompression procedures of the area with necrosis, associated or not with free or pediculated cortical-spongy grafts. As the disease advances and changes in articular congruence take place, procedures such as osteotomies, hemiarthroplasties or unicompartmental arthroplasties may be indicated; in the most advanced cases, the solution is total arthroplasty [14].

\section{The Carpal Tunnel Syndrome}

The occurrence of carpal tunnel syndrome has been reported among the osteoarticular changes observed in HIV/ AIDS patients receiving therapy, although the existing reports question its higher incidence in these patients compared with the general population $[17,18]$. Incidence in the general population was found to be around $3.8 \%$ during clinical evaluation and around $2.7 \%$ when electroneuromiography was used. In the tested HIV-positive population, incidence was maintained very close to that found in the general population [17].

This syndrome has been related to the use of highly active antiretroviral therapy, especially protease inhibitors, and would result from the already known metabolic disorders of myxedematous material deposited in the carpal tunnel, with consequent nerve compression. Other factors are associated with the development of this syndrome in HIV/AIDS patients, such as labor activities, hypothiroidism, hyperglicemia, rheumatoid arthritis, obesity and miscellaneous metabolic disorders. Therefore, direct correlation with the presence of HIV and antiretroviral therapy is still controversial [17,18].

To diagnose the carpal tunnel syndrome, the following criteria must be met: sensitive disorder in the median nerve territory, positive Tinel's sign and positive Phalen's sign. The sensitivity and specificity of these combined criteria exceed $90 \%$, as confirmed in patients participating in the electroneurophysiological study.

The treatment is based on compression syndrome staging. Treatment during the mild stage is conservative and consists in using night braces and anti-inflammatory drugs [19]. During moderate to severe stages, surgical treatment is indicated. This can be performed either in the conventional way or endoscopically $[20,21]$. In both procedures the median nerve is decompressed through the opening of the flexor retinaculum.

\section{Adhesive Capsulitis}

Adhesive capsulitis has been associated with HIV-patients treated with an antiretroviral regimen including protease inhibitors [22]. The cases reported in literature are limited to shoulder involvement, suggesting that other sites are rarely involved [23,24]. Typical symptoms of the condition include shoulder unilateral/bilateral progressive pain with restriction of both active and passive arc of motion. Usually the onset of symptoms is insidious and occurs 12 to 14 months following the beginning of antiproteases [22]. Ordinary X-ray can show bone rarefaction caused by lack of use, although 
Figure 1.

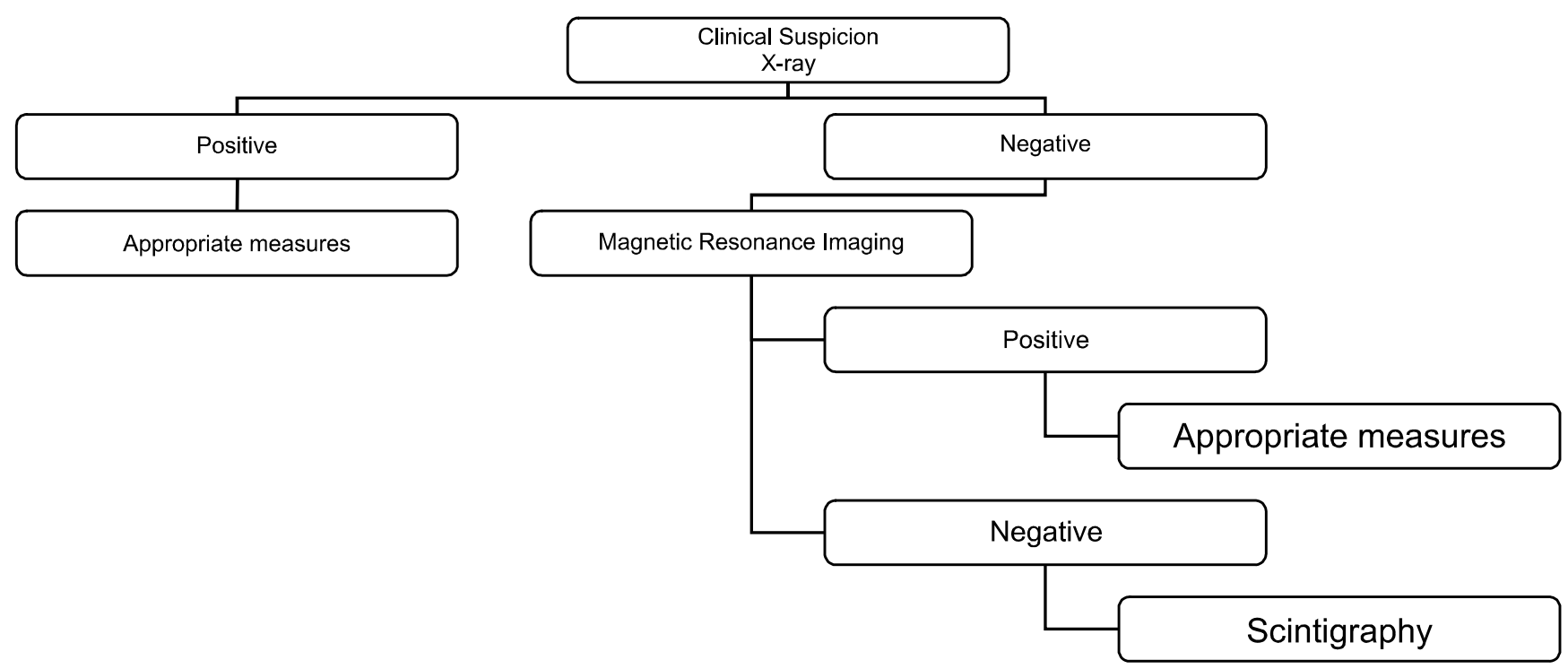

Table 1. Ficat-Arlet Classification

\begin{tabular}{clll}
\hline Stage & Symptoms & X-ray & Scintigraphy \\
\hline 0 & None & Normal & Reduced capture \\
1 & None to mild & Normal & Reduced capture \\
2 & Mild & Change in density & Increased capture \\
2A & & Sclerosis or cysts & \\
2B & & Applanation (crescent sign) & \\
3 & Mild to moderate & Loss of sphericity & Increased capture \\
4 & Moderate to severe & Reduction of the articular space & Increased capture \\
& and/or acetabular changes & & \\
\hline
\end{tabular}

arthromagnetic resonance is the evaluation of choice for diagnosis. Symptoms tend to regress spontaneously after a 6-24-month period if an appropriate treatment is instituted and the antiretroviral regimen is suspended [22].

Treatment of adhesive capsulitis depends on its evolution time and the severity of the adherences. In milder cases, conservative treatment with analgesics and anti-inflammatory agents plus physical therapy is the most indicated [25]. In more severe cases that do not respond to conservative treatment, the arthroscopic treatment with anterior and posterior capsulotomy to improve the arc of motion, mainly abduction and external rotation, is currently the most indicated, followed by early mobilization. We have avoided the indication of isolated manipulation due to the higher incidence of fractures in the proximal third of the humerus and the fact that the postoperative pain is more intense, making early mobilization difficult [26].

\section{References}

1. Amorosa V., Tebas P. Bone disease and HIV infection. Clin Infect Dis 2006; $42: 108-14$.
2. Bruera D., Luna N., David D.O., et al. Decreased bone mineral density in HIV-infected patients is independent of antiretroviral therapy. AIDS 2003; 17:1917-23.

3. Delaunay C., Loiseau-Peres S., Benhamou C.L. Osteopenia and human immunodeficiency virus. Joint Bone Spine 2002;69:105-8.

4. Dolan S.E., Huang J.S., Killilea K.M., et al. Reduced bone density in HIV-infected women. AIDS 2004;18:475-83.

5. Seminari E., Castagna A., Soldarini A., et al. Osteoprotegerin and bone turnover markers in heavily pretreated HIV-infected patients. HIV Med. 2005;6:145-50.

6. Jain R.G., Furfine E.S., Pedneault L., et al. Metabolic complications associated with antiretroviral therapy. Antiviral Res 2001;51:151-77.

7. Mondy K., Tebas P. Emerging bone problems in patients infected with human immunodeficiency virus. Clin Infect Dis 2003;36(Suppl 2):101-5.

8. Jain R.G, Lenhard J.M. Select HIV protease inhibitors alter bone and fat metabolism ex vivo. J Biol Chem 2002;277:19247-50.

9. Mora S., Sala N., Bricalli D., et al. Bone mineral loss through increased bone turnover in HIV-infected children treated with highly active antiretroviral therapy. AIDS 2001;15:1823-9.

10. Mora S., Zamproni I., Beccio S., et al. Longitudinal changes of bone mineral density and metabolism in antiretroviral-treated human immunodeficiency virus-infected children. J Clin Endocrinol Metab 2004;89:24-8. 
11. Tan B.M., Nelson R.P Jr., James-Yarish M., et al. Bone metabolism in children with human immunodeficiency virus infection receiving highly active antiretroviral therapy including a protease inhibitor. J Pediatr 2001;139:447-51.

12. Parsonage M.J, Wilkins E.G.L., Snowden N., et al. The development of hypophosphataemic osteomalacia with myopathy in two patients with HIV infection receiving tenofovir therapy. HIV Med 2005;6:341-6.

13. Mahoney C.R, Glesby M.J., DiCarlo E.F., et al. Total hip arthroplasty in patients with human immunodeficiency virus infection. Acta Orthop 2005;76:198-203.

14. Allison G.T., Bostrom M.P., Glesby M.J. Osteonecrosis in HIV disease: epidemiology, etiologies and clinical management. AIDS 2003; 17: 1-9.

15. Allen S.H., Moore A.L., Tyrer M.J., et al. Osteonecrosis of the knee in a patient receiving antiretroviral therapy. Int J STD AIDS 2002;13:792-4.

16. Dutkosky J. Miscellaneous nontraumatic disorders. In: Canale ST, editor. Campbell's Operative Orthopaedics. 10th ed. Philadelphia: Mosby; 2003. p. 946-950.

17. Sclar G. Carpal tunnel syndrome in HIV-1 patients: a metabolic consequence of protease inhibitor use? AIDS 2000; 14:336-8.

18. Asensio O., Caso J.A.A., Rojas R. Carpal tunnel syndrome in HIV patients? AIDS 2002; 16:948-50.
19. Ucan H., Yagci I., Yilmaz L., et al. Comparison of splinting, splinting plus local steroid injection and open carpal tunnel release outcomes in idiopatic carpal tunnel syndrome. Rheumatol Int 2006;27:45-51.

20. Zyluk A., Strychar J. A comparison of two limited open techniques for carpal tunnel release. J Hand Surg Br 2006;31:466-72.

21. Atroshi I., Larsson G.U., Ornstein E., et al. Outcomes of endoscopic surgery compared with open surgery for carpal tunnel syndrome among employed patients: randomized controlled trial. BMJ 2006; $332: 1473$.

22. De Ponti A., Vigano M.G., Taverna E., et al. Adhesive capsulitis of the shoulder in human deficiency virus-positive patients during highly active antiretroviral therapy. J Shoulder Elbow Surg 2006; 15 : $188-90$.

23. Atalay A., Ozdemir O., Guven G.S., et al. HIV infection and shoulder pain: a challenging case. Rheumatol Int 2006;26:680-2.

24. Tehranzadeh J., Ter-Oganesyan R.R., Steinbach L.S. Musculoskeletal disorders associated with HIV infection and AIDS. Part II: Non-Infectious musculoskeletal conditions. Skeletal Radiol 2004;33:311-20.

25. Shaffer B., Tibone J.E., Kerlan RK. Frozen shoulder. J Bone Joint Surg Am 1992;74:738-46.

26. Warner J.J.P., Allen A., Marks P.H., et al. Arthroscopic release for chronic refractory adhesive capsulitis of the shoulder. J Bone Joint Surg Am 1996;78:808-16. 